\title{
The Maker Movement and the Disruption of the Producer-Consumer Relation
}

\author{
Elisabeth Unterfrauner ${ }^{1}$, Christian Voigt ${ }^{1}$ \\ Maria Schrammel ${ }^{1}$ and Massimo Menichinelli ${ }^{2}$ \\ ${ }^{1}$ Centre for Social Innovation, Technology and Knowledge, 1150 Vienna, Austria \\ ${ }^{2}$ IAAC I Fab City Research Lab, 08005 Barcelona, Spain \\ unterfrauner@zsi.at \\ voigtezsi.at \\ schrammelezsi.at \\ massimo@fablabbcn.org
}

\begin{abstract}
The Maker movement represents a return of interest to the physical side of digital innovation. To explore expectations and values within the Maker movement, we applied qualitative research method, interviewing 10 managers of maker initiative as well as 39 makers from eight different countries. The paper analyses how the Maker movement is contributing to a change in production, logistics and supply chains and how it changes the relationship between producer and consumer. Based on the interview data and supported by literature, the study indicates that the Maker movement has the potential to impact producer-consumer relationships in many ways. Making, on a bigger scale would mean producing locally, de-centralised and on-demand. This would have an impact on the logistics and the supply chain. Long transportation routes would be avoided and shorter supply chains would make some of the-inbetween vendors obsolete. Makers as prosumers, who produce for themselves, are introducing two growing phenomena: a more personalised relationship between maker and object and personalised products as a form of self-expression.
\end{abstract}

Keywords: Maker Movement, Digital Social Innovation, Supply Chain, Prosumer, Sharing Economy.

\section{The Maker Movement and Digital Social Innovations}

Broadly speaking, the Maker movement represents a return of interest to the physical side of innovation following an almost exclusive focus on the digital side. It thus bridges the gap between the digital and physical world, between the peer collaborative platform for working on and sharing of designs and the physical fabrication in the maker space or fablab or even at home: Neil Gershenfield, one of the godfathers of the Maker movement, described this evolution as the connection between bits and atoms based on digital fabrication technologies which are "an evolving suite of capabilities to turn data into things and things into data" leading to "manufacturing processes in which the materials themselves are digital" [1]. 
The Maker movement can be described as Digital Social Innovation (DSI) as it follows some of the typical characteristics of DSI [2, 3, 4]: e.g. Lowering entry barriers to innovation due to fast prototyping and experimenting of ideas; enabling collaborative working physically and online on a multitude on maker platforms; making community knowledge greater than individual knowledge as makers are keen on the value of openness and share their knowledge.

We depart from the premise that information systems impact our social and working life in multiple ways and although in an organisational context the focus lies often on economic aspects, issues around environmental or social implications come to the fore more strongly, as shown in recent debates about technologies' energy consumption [5] or changing labour markets due to on-going automations in the context of industry 4.0 [6]. Hence, looking at maker technologies through the lens of information systems research, Churchman's [7] call for reflective and systemic systems designs is more appropriate than ever. Churchman emphasised the practical nature of reflectivity with a simple example of an information system that increases the output of cigarettes, obviously being an improvement in the eyes of the client. Yet, so Churchman, this does not exempt the information systems designer from critically reflecting upon who else is affected by these 'improvements', a deliberation that could lead to the realisation that the same information system is likely to also increase costs for the national health care system for example (ibid.). Transferring this analogy to the discussion of maker technologies, we see a need for maker spaces to develop a stronger awareness of their role in areas not yet central to the Maker movement, such as environmental protection, waste reduction, energy saving or re-use of materials.

The aim of this paper is to investigate one of the potential future impacts of the Maker movement that affects not only makers but the society at large if the Maker movement keeps growing in this fast pace [8], i.e. the disruption of supply chains.

\section{Cross-Case Analysis of Maker Initiatives}

This study is part of a vast cross-case analysis of maker initiatives that comprised a range of research questions under three broader research pillars. While the overall study generated insights on how maker initiatives are organised, how makers develop their skills and how they collaborate and which values are created in the Maker movement, this study concentrates on one particular topic.

To explore the expectations and values of making, we applied qualitative research methods. Unlike quantitative research, which tests already formulated hypotheses and requires certain conditions such as representativeness of the data and validity of test items, qualitative research methods are best used to explore emerging concepts, where statistical data is not yet available [9]. 
This has also the advantage to be able to go into more depth and follow up on topics that seem rich of interpretations. For this purpose, we developed a semistructured interview guideline, i.e. a set of interview questions that can be used in a flexible manner while still preserving coverage of similar topics across multiple interviews. In other words, the interviewer was requested to ask all the questions but was free to add additional ones in case a topic should have been explored in more detail. The interview was divided in three big sections, questions regarding the governance and organisation of maker communities, regarding peer and collaborative behaviours among makers and lastly, the value and impact of making and contained [15].

The interviews were all recorded and then transcribed to allow for detailed content analysis (e.g. labelling of text snippets according to a coding scheme). In the coding process the researcher is going through the interview material with pre-defined codes and identifying new topics at the same time [10].

We chose 10 maker initiatives in 8 countries, which were very different in nature, from a Mini Maker Faire to fablabs and maker spaces that were independent or part of a university: Happylab Vienna, Austria; DTI lab, Denmark; Fablab Barcelona, Spain; Officine Arduino, Italy; HRW Lab, Germany; Dezentrale, Germany; Mini Maker Faire Tartu, Estonia, Fablab Zagreb; Croatia; Smart Bending Factory, the Netherlands; and Create It Real, Denmark.

The maker initiatives were selected based on the mapping on two dimensions, where we wanted to achieve the most possible diversity (c.f. figure 1). The scale and interaction dimension ranges from Single to Network, i.e. from makers who work on their own and would be characterised by situational awareness as they act on their individual preferences and needs and are not part of an initiative to a network of strongly connected maker initiatives with distributed awareness, where glocal solutions are found and shared. The second dimension was the CAPS (Collective Awareness Platforms for Sustainability and Social Innovation) social innovation dimension that spans from social demand to systemic change. 


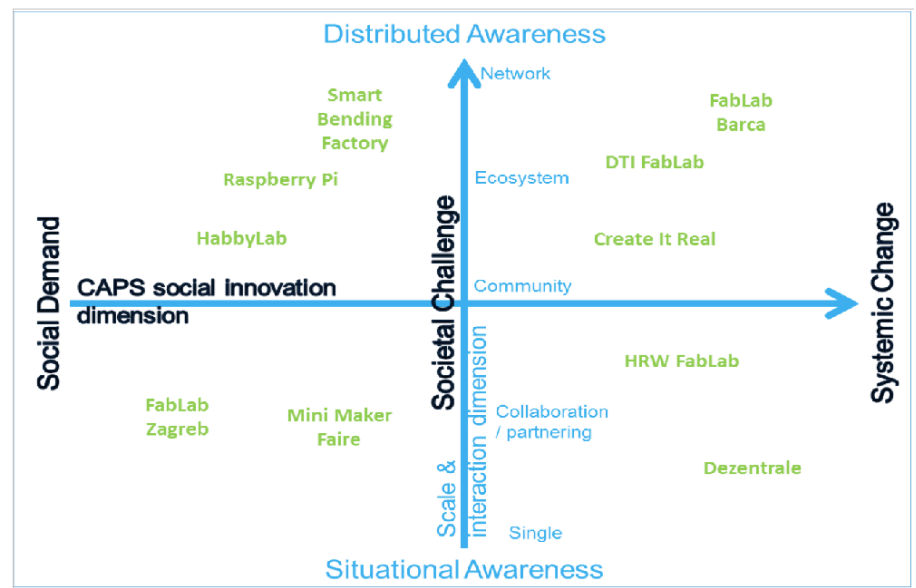

Fig. 1. Scale/social innovation dimension [11]

To familiarise with the maker world, we describe in the following one maker initiative and one maker product in particular.

Happylab Vienna is registered as a Fablab. Fablabs are places that encourage collaborative learning, knowledge sharing and changing the local economy for the better. Moreover, Fablabs aim to provide access to digital fabrication tools for all and subscribe often to a philosophy of openness as a core value (e.g. open access, open designs, open source). Happylab's business model is based on membership fees as well as income from specific workshops. After having received a free training on the machines, Fablab members are allowed to use them, i.e. 3D printers, laser cutters, milling machines, computers, etc. [22].

An example of a maker product that has been created as a first prototype in Happylab is the Wadi tool (c.f. Figure 2). It is used for water purification, it detects when water is safe to drink after its exposition to the sun. After having created and tested the prototype in the maker space, the maker sought for funding and was finally able to produce in bigger quantities and has founded his own company, selling now Wadi tools worldwide, especially in developing countries. 


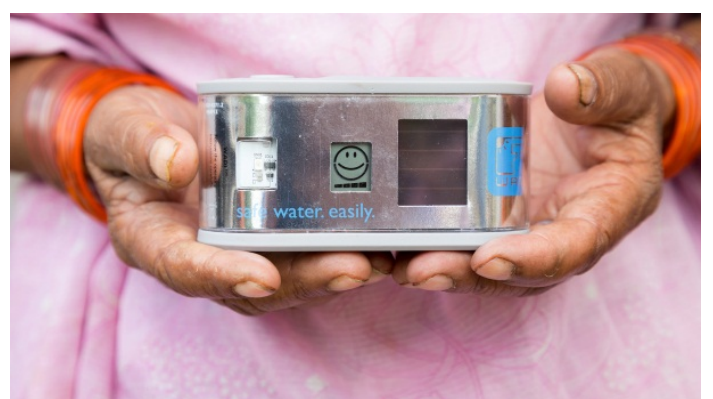

Fig. 2. Wadi for water purification via solar energy, developed by Helioz (https://www.trendingtopics.at/wadi-who/)

To answer our pre-defined research questions, we interviewed the managers of the different maker initiatives and asked them to point us to 3 makers resulting in 39 interviews in total (one maker interview was not feasible). Since we wanted to collect the data from as diverse makers as possible in terms of regions and types of makers, we targeted one female maker, one maker with commercial and one maker with social ambitions to get diverse answer patterns, reflecting the conditions of making under different circumstances. The transcript of the interviews were analysed qualitatively [12] following a deductive and inductive coding approach. The deductive codes applied were derived from the research questions, whereas inductive codes directly evolved from the interview data allowing for the unexpected [13]. The interviews took place either face-to-face or via telephone or Skype between September and November 2016. The age of the interviewees ranged from around 20 and 50 years and reflects the maker population [14].

This paper focuses on the perceived and aspired impact makers consider the Maker movement and digital fabrication technologies have on the supply chain of goods and patterns of consumption.

Specifically, key research questions addressed in this paper are:

- In what ways do maker communities change production, logistics and supply chains?

- How does making change the relationship between producer and consumer?

The clustered topics that emerged in the inductive coding process (following the deductive coding process where we looked for "change production, logistics and supply chains" and "impact on relationship between producer and consumer") are the following:

1. Change from mass production to decentralised production

2. Development cycle and prototyping

3. Personalisation and customisation

4. Circular economy and repair culture

5. Mentality shift - from consumers to prosumers 


\subsection{Change from Mass Production to Decentralised Production}

Many interviewees saw a change from mass production to distributed and decentralised production due to the Maker movement taking place, from industry products that are centrally produced and transported worldwide via a chain of in-between vendors and with complex logistical systems to decentralised production with on-demand products that are locally produced and with shorter ways of transportation. For some this is just the logic conclusion: "The more people do themselves, the less you need mass production" (maker, Italy).

This evolution can be seen in the light of similar trends such as the growing consciousness of consumers in general about any kind of product that they purchase, be it food that has to be organic and have a low carbon footprint, textiles that are produced under fair conditions, etc. [16]. One of the interviewees made a link to a wider historical context, an evolution that bring us somehow 'back to the roots': "It started with very decentralised production units, meaning small villages. People have produced their own things here, but there was little exchange with the outside world. (...) At some point, in the context of industrialisation, there was a very strong centralisation of production processes. (...) In the beginning we had an analogous decentralised society and then we had an analogous centralised society. By now we have a digital centralised society and the step which now has to happen is a digital decentralisation" (maker, Germany). This turn from centralised to decentralised production has an impact on labour and employment. Mass production has been made possible because of division of work and expertise, "products get made in a company where there is a whole team of designers and engineers working on just one object of the whole, and then there are the engineers working on the mechanical objects, and then together they create the product. There is a team of different specialists (...) whereas in this case (as a maker) I work alone (...) you have to be a specialist in every field, which I am obviously not, so I have to learn little by little" (maker, Spain). From an environmental perspective, on the one hand, mass production is associated with producing more waste, and consuming more energy, not only in the production of the products but also in their logistics. Huge batches of products are produced, stored somewhere and transported to their customers and when they are out-dated they are thrown away (maker, Italy). Making on the other hand allows for decentralised production, which reflects the ambition for sustainability that the Maker movement is keen of (maker, Germany). As one manager points out (SBF), makers make smarter use of their raw materials, produce less waste and have shorter transportation routes.

According to the interviewees, decentralised production means producing small batches, probably just for the own consumption, either at home or in a maker space. Since all have the possibility to access digital fabrication tools it is not necessary to buy industry products and support the traditional supply chain but to disrupt it by producing and consuming locally [17]. Opposed to mass production where big batches are produced based on a more or less rough estimation of the market need, on-demand production means producing products just for customers who have already ordered 
the product, as makers described in the interviews. Thus, with on-demand production overproduction is avoided with reduced production of waste. Maker communities are taking advantage of the many platforms where they can share and sell their design and produce on demands: "I created an Etsy page, it is an online shop where you can upload a picture of your design and people can see what you are selling" (maker, Estonia).

Local production has evolved with the Maker movement because technologies that were formerly available only to industries can now be accessed by anybody. The technologies develop in fast pace, get cheaper and smaller and also production costs decrease and thus the trend to produce locally might increase according to a maker in Barcelona (maker, Spain): "It is kind of a natural evolution going in that direction, it will definitely be a large part of economy to have local manufacturing and a different style of production versus mass production". This view was congruent with statements of many other makers. One maker from Denmark sees this trend having a bigger impact in the near future, i.e. 5 to 7 years. He imagines that more people will become makers and access maker spaces to produce things for their own consumption.

Decentralised production works already well for niche products, on-demand products, for customised and personalised products. In contrast to these kind of products, objects that are being produced in bigger quantities cannot be produced in a maker space because the production price would not pay off and the production cycle would just not be feasible: "If you are producing small scale it makes sense to produce in a maker space but once you want to produce bigger batches and you want to have a higher revenue you cannot do that anymore in a maker space" (maker, Germany).

Opposed to these rather visionary opinions of makers described above, other makers expressed their concerns and were quite sceptical. One maker from Germany, for instance, saw the disruption of supply chain taking place not in the provision of the final product but in the step before that, i.e. in prototyping.

\subsection{Development Cycle and Prototyping}

Many makers see the big advantage of maker spaces in fast prototyping. It is possible to test own ideas with very little expenditures. For instance, instead of having to produce an injection mould already for the first prototype, which can be as expensive as 30.000 euro, makers can produce a prototype in the maker space and test it before going into the production of bigger batches. This allows also for faster changes in design or adaptation in the functionality of their prototypes according to the test results and their experience with the first prototypes: "This is so great, because you can change your design very quickly or try out something new, also just with 3 pieces. This works very well and it results in a top-quality" (maker, Germany). These advantages, i.e. convenient production of prototypes and agile adaptation at any point 
during the testing phase, allow for a shorter and faster development cycle compared to the traditional development cycle of a product. For instance a maker in Germany observed: "When others see how fast a prototype can be developed, then they are at times shocked. (...)This makes you realise that a lot will change in this area. Especially the development of prototypes works a lot faster with these things". Also the maker is in control of every step in the production of the prototype, which is a big advantage. Basically all makers and managers agreed that the Maker movement had an impact on the supply chain regarding the development of prototypes (a few saw the change happening on a bigger scale, i.e. disrupting the entire supply chain- from mass to local production - as described above). On the long run, industries might benefit from this evolution: "These maker-spots are places for creating prototypes. They are unique”. Industries with their injection moulding machines produce batches of 5.000 to 10.000 pieces "and we produce only single pieces. We could not produce that many in a short amount of time in an efficient manner. The only thing we could do is to contribute to a change of the production chain. In a way we say: 'Yes, we found a better example. A better prototype, which functions better.'” (maker, Germany).

\subsection{Personalisation and Customisation}

A big advantage of making is the opportunity for personalisation and customisation of products [18]. Most of the interviewed makers and managers shared this view. With digital fabrication technologies it is relatively convenient to make individual, personalised and customised products since production time and costs do not increase as much, whereas with mass production this would be impossible. It is easy to modify a design that is already available, and to give it a personal touch (maker, Spain). There are millions of designs available on different platforms that can be downloaded and customised (maker, Germany). This then opens up the debate as to how to protect ones designs and also give credit to designers if a design is taken from someone else and changed according to the other person's needs.

Customers want to have customised products: "People will want different things [than] they want today. They won't settle for the same glasses [as] their neighbours, they want their glasses, which fit them perfectly and have the right shape for their ears. Every single product that they will want will have to be personalised" (manager, Denmark). Besides, "The world is becoming more and more colourful, and more unique demands for products increase" (maker, Estonia). Customised products might replace on-the shelf products since they reflect the individual needs better. For instance, a maker who was dissatisfied with the light-holder of his bike, and who failed to find a suitable replacement on the market, designed and created his own, personalised light holder in the maker space (maker, Denmark). Personalised objects hold different values for customers: "If you have a project that you know has been made specifically for you, not just because you ordered it but because you know where it's been made, perhaps you have been there, you know people, who made it, and maybe 
you have modified the file (...) you have a closer personal relationship to that object, and you'll treat that object in a different way because of it" (maker, Spain).

\subsection{Circular Economy and Repair Culture}

In respect to the disruption of supply chains many makers recognize that the Maker movement contributes to a circular economy model [19]. As described in this model, makers are aware of waste production and are keen on resource efficiency and renewable resources, in comparison to the linear model), which follows a 'take, make, dispose' thinking (c.f. Figure 3).

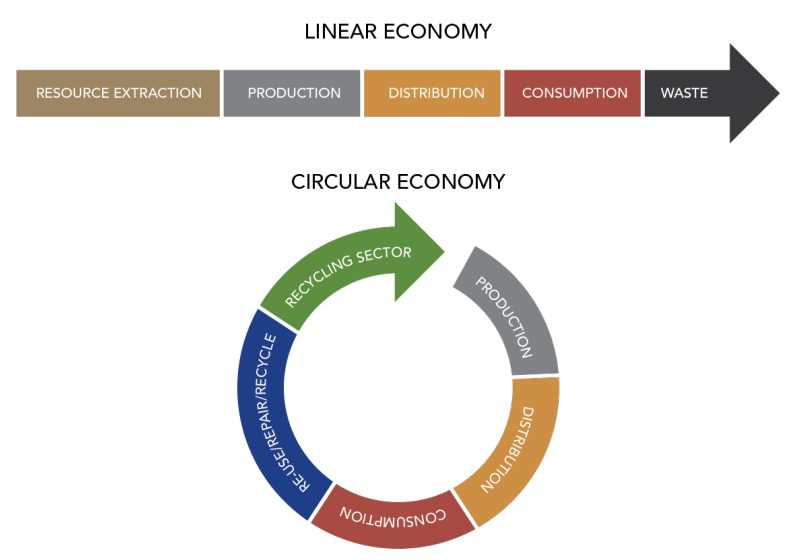

Fig. 3. Linear versus circular economy ${ }^{1}$

Resulting terms used by the makers and/or managers during the interviews comprised of: re-cycling, up-cycling, and repair culture. Making in essence supports the longevity of products via recycling of industrially produced that would otherwise be thrown away because substitute parts are no longer available. According to a manager in Denmark: "There is much value behind the 3D printer because you can replace the world around you and you can improve it and create your own parts". Up-cycling or the re-assembling of parts in pre-produced products or making use of pre-produced product parts in ways different from its original design, by adapting and making re-configurations on the object occurs frequently within the movement. "Upcycling is a big issue in the Maker movement (...). You take something old and turn it into something new. This could be a lot of innovative approaches for the future" (maker, Germany). This allows for a continued use of the object and thus contributes to the circular economy with smaller loops in contrast to bigger loops "in

https://www.weforum.org/agenda/2016/05/can-the-circular-economy-transform-theworld-s-number-one-consumer-of-raw-materials/ 
which you shred everything and try to spate it, which takes a lot more effort" (maker, Germany).

\subsection{Mentality Shift - from Consumers to Prosumers}

In the future, the impact of the Maker movement on supply chains will be considerable, many makers believe. However, a mentality shift with consumers becoming producers and being more aware of consumption is a condition for this major change to happen. Societal values are slow to change and sometimes it takes several generations to think about things differently, act differently and create the desired lasting impact [20].

Makers appear to take a dim view of consumerism, especially in light of their increased awareness and desire to know and understand how things are made/produced and to be in control of the production process. Creating individual products means turning away from consumerism (maker, Germany). According to a maker in Barcelona: "the current consumerism model is also in a way detached from the consciousness of how things are made because everything is done in the industry and we are losing the crafts. So we don't know how a bottle of water is made; we don't know what it implies in resource and in time. We don't see the footprint of an object, and I think this is very important to understand this" (maker, Spain).

The manager of Fablab Zagreb, for instance, is convinced that approaching younger generations who are creative and have not yet become consumers, and showing them how to make things, supports a paradigm shift that disrupts this relationship between producer and consumer. Similarly, the CIR manager agrees with that: "It is a change that will take a bit of time, because you have to start with a new generation and teach them it doesn't have to be like this (that you purchase things), and they can be picky, and they can influence their design and be a designer".

A maker in Estonia pointed out that during the communist period people had to make many things on their own but today, moving back a little towards doing more things could have a positive impact on the psychology of people (maker, female, AHHAA).

Moreover, the paradigm shift that is slowly taking place, is people driven (as the Maker movement per se), in that sense a bottom-up approach and thus does not have to be steered by politics and policy makers (maker, Spain). Producing for the individual consumption and producing locally would probably have an impact on the pricing of products. One maker noted that decentralised production would have to be geared towards profit (maker, Germany) and would not have to follow a tradition business model.

While transportation costs and logistics would be reduced, production costs would increase but customers may also be willing to pay more knowing where, how 
and by whom a product has been made. There is something wrong with the system, according to a maker from Germany, if imported products are much more convenient than locally produced products (maker, Germany)

\section{Conclusions}

This paper investigates the potential future impacts of the Maker movement in the disruption of supply chains and the consequent impact on society and in social innovations. Several clustered topics were found in the inductive coding process of the interviews, pointing to five current emerging trends:

1. Change from mass production to decentralised production: a local and small scale on-demand production that will change labour, employment and reduce the impact on the environment.

2. Development cycle and prototyping: cheaper and faster processes that impact design activities and consequently also manufacturing systems.

3. Personalisation and customisation: activities based on openly accessible designs and the high personal value that exceeds production time and costs.

4. Circular economy and repair culture: re-cycling, up-cycling, repair activities based on a new awareness.

5. Mentality shift - from consumers to prosumers: a slow and future impact emerging from the interest in understanding manufacturing processes that will bring a critical view of consumerism.

Coming back to the first research questions, i.e. in what ways do communities in the Maker movement change production, logistics and supply chains, based on the interview data, it has to be answered that the Maker movement has the potential to impact on in many ways. Making on a bigger scale would mean producing locally, de-centralised and on-demand. This would have an impact on the logistics and the supply chain. Long transportation routes would be avoided; the supply chain would be shorter and make some of the-in-between vendors obsolete. A local and on-demand production would have a positive environmental impact because over-production would be avoided and less logistics would be needed to handle end-products, instead logistics would be required for storage and distribution of raw materials. Probably some sectors would be in danger while others would flourish if decentralised production grew and would replace centralised production as makers and maker spaces managers argued. They imagined that boundaries between professional and amateur designers could be increasingly less clear and therefore change established dynamics. Marketing and distribution could be replaced by platforms (that are already available now), where makers share their design and their customers can order products that can be manufactured with digital fabrication technologies or downloaded for local production. This would ultimately result in the creation of new jobs in the regions 
with less out-sourcing to other countries and thus it would have a tremendous economic impact regionally but also globally since some of the economic forces would be shifted. For instance, in the region craftsmen would play an increasingly important role in the maker community.

Making would have an impact on circular economy because the longevity of products would be supported through the repair culture the Maker movement holds on to by up-cycling and recycling. This future scenario however has many limitations. Most makers agree that there is a real potential for all the above described impacts but currently the impact is taking place rather on low-scale level. The condition for a bigger scale is a mentality shift among consumers, who need to demand products of any kind to be locally produced or which could be produced by themselves. Currently the Maker movement has already an impact on the development cycle of prototypes. Thanks to making, prototypes can be produced faster and cheaper, ideas can be tested easier before investing a lot of money. Even if making and decentralised production might never completely replace centralised production it is already taking shape for certain kind of products: niche products, personalised products, customised products and products in small quantities.

The second research question, i.e. how does the Maker movement change the relationship between producer and consumer, can be answered as follows based again on the maker and maker space manager interviews. Making definitely has an impact on the relationship between producer and consumer. Makers who produce for themselves can be considered prosumers because producing and consuming is merged in their activities. Making an object has an impact to the relation towards this object because it has been specifically been made for that person Probably this object would be treated differently compared to an object that is bought from the shelf. Consumers of maker objects are believed to have a different awareness of products in general and many interviewed makers showed an anti-consumerism attitude. Makers want to know how products are produced, what is inside an object, and open up "black boxes". Some argue that they would like to be in control of the production steps because buying off the shelf means missing out on the different production steps and losing that knowledge. So this growing desire to produce regionally, environmentally friendly and sustainable can be seen in light of a trend that is moving somewhat "back to the roots", where villages were autarchic, with strong regional consciousness.

The interviews were structured in order to understand the dimension of social innovation in the transformation of supply chains; from these interviews we suggest these guidelines for facilitating, improving and scaling such dynamics on digital platforms:

- Change from mass production to decentralised production: platforms could facilitate the organization of a local, small scale and on-demand production by locally connecting makers with suppliers, maker spaces, manufacturers and consumers. 
- Development cycle and prototyping: platforms could provide tools that facilitate the organisation of design and prototyping processes.

- Personalisation and customisation: platforms could provide tools that facilitate the collaborative development, organization, sharing, commercialisation, management and customisation of openly accessible designs.

- Circular economy and repair culture: platforms could measure and visualise the impact of Maker initiatives on supply chains and the environment in order to build awareness and improve participation.

- Mentality shift - from consumers to prosumers: platforms could focus on communicating the values of Maker initiatives and on visualising manufacturing processes and the impact of both consumerism and Maker initiatives on them.

This research explored expectations and values within the Maker movement and provided an overview of how makers believe their activities will change existing production practices, organisations and networks. Further research might be done in order to uncover the philosophical and political dimensions of such values and expectations; less in-depth but more large-scale inquiries might provide an overview of the geographical and social distribution of such beliefs, and if these are homogenous or if there are geographical or social differences. This would help us understand more the various motivations for participation in the Maker movement and maker initiatives, in turn providing further insights on digital platform development by providing guidelines for improving the engagement of makers. Furthermore, these beliefs could be compared with what established business and research initiatives believe about the Maker movement in order to understand how the Maker movement is perceived; finally, how these values and expectations informs the impact of the Maker movement is an important topic to be investigated in order to understand if and what impact it has on society and the economy, and how specific values inform such impact.

Acknowledgements: The study has been conducted with the support of the European Commission in the framework of the Horizon2020 Project MAKE-IT (GA 688241). For further information: www.make-it.io

\section{References}

1. Gershenfeld, N.: Bits and Atom. Presented at the 2005 International Conference on Digital Printing Technologies (2005).

2. Voigt, C., Montero, C.S., Menichinelli, M.: An empirically informed taxonomy for the Maker Movement. In: International Conference on Internet Science. pp. 189204. Springer (2016).

3. Bria, F., Almirall, E., Baeck, P., Halpin, H., Kingsbury, J., Kresin, F., others: Digital social innovation interim report. Lond. Nesta. (2014).

4. Unterfrauner, E., \& Voigt, C. (2017). Makers' ambitions to do socially valuable things. The Design Journal, 20 (Sup1) 
5. Watson, R.T., Boudreau, M.-C., Chen, A.J.: Information systems and environmentally sustainable development: energy informatics and new directions for the IS community. MIS Q. 23-38 (2010).

6. Brettel, M., Friederichsen, N., Keller, M., Rosenberg, M.: How virtualization, decentralization and network building change the manufacturing landscape: An industry 4.0 perspective. Int. J. Mech. Ind. Sci. Eng. 8, 37-44 (2014).

7. Churchman, C.W.: Philosophical speculations on systems design. Omega. 2, 451465 (1974).

8. Dougherty, D.: The maker movement. innovations. 7, 11-14 (2012).

9. Silverman, D.: Qualitative Research. SAGE (2016).

10.Flick, U.: An Introduction to Qualitative Research. SAGE (2014).

11. Millard, J.: D2.2 Monitoring and assessment framework. (2016).

12.Mayring, P.: Einführung in die qualitative Sozialforschung: Eine Anleitung zu qualitativem Denken. Beltz Verlag, Weinheim und Basel (2010).

13. Reichertz, J.: Abduktion, Deduktion und Induktion in der qualitativen Sozialforschung. In: Flick, U., von Kardoff, E., and Steinke, I. (eds.) Qualitative Forschung. pp. 276-285. Rowohlt Taschenbuch Verlag, Hamburg (2012).

14.Bianchini, M., Menichinelli, M., Maffei, S., Bombardi, F., Carosi, A.: Makers' Inquiry. Un'indagine socioeconomica sui makers italiani e su Make in Italy. Milano: Libraccio Editore. Retrieved from http://makersinquiry. org (2015).

15.Unterfrauner, E., Schrammel, M., Hofer, M., Fabian, C.M., Voigt, C., Deljanin, S.R., Sorivelle, M.N., Devoldere, B., Haga, H.: Final case study report focusing on cross-case analysis. (2017).

16.Hughner, R.S., McDonagh, P., Prothero, A., Shultz II, C.J., Stanton, J.: Who are organic food consumers? A compilation and review of why people purchase organic food, (2007).

17.Kohtala, C.: Addressing sustainability in research on distributed production: an integrated literature review. J. Clean. Prod. 106, 654-668 (2015).

18.Osunyomi, B.D., Redlich, T., Buxbaum-Conradi, S., Moritz, M., Wulfsberg, J.P.: Impact of the Fablab Ecosystem in the Sustainable Value Creation Process. (2016).

19.Lacy, P., Rutqvist, J.: Waste to Wealth: The Circular Economy Advantage. Springer (2016).

20.Joore, J.P.: New to Improve - The Mutual Influence between New Products and Societal Change Processes. JP Joore (2010).

21.Nascimento, S.: Critical notions of technology and the promises of empowerment in shared machine shops. J. Peer Prod. 5, (2014).

22.Voigt, C., Unterfrauner, E., \& Stelzer, R. (2017). Diversity in FabLabs: Culture, Role Models and the Gendering of Making. In International Conference on Internet Science. Greece. 
